\title{
Meta-Analysis of the Clinical Effect of Acupuncture on Postherpetic Neuralgia
}

\author{
Lu Wang1, Zhenlin Chen ${ }^{1 *}$, Shuo Zhang ${ }^{2}$, Wenjing Ma1, Tiantian Gao ${ }^{1}$ \\ ${ }^{1}$ Shaanxi University of Chinese Medicine, Xianyang 712046, Shaanxi Province, China \\ ${ }^{2}$ Beijing University of Chinese Medicine, Beijing 100029, China \\ *Corresponding author: Zhenlin Chen, chenzhenlin19972@163.com
}

Copyright: (C) 2022 Author(s). This is an open-access article distributed under the terms of the Creative Commons Attribution License (CC BY 4.0), permitting distribution and reproduction in any medium, provided the original work is cited.

\begin{abstract}
Objective: To systematically evaluate the safety and efficacy of acupuncture on patients with postherpetic neuralgia . Methods: Based on the heyword retrieval method, China National Knowledge Infrastructure (CNKI, 1978-2020), China Biomedical Literature database (CBM, 1979-2020), VIP database (WEIPU, 1989-2020), Wanfang database (19892020), Cochrane Library, PubMed, and Embase were searched; randomized controlled trials of the use of acupuncture in the treatment of postherpetic neuralgia were screened out; the quality of the included literatures was evaluated based on the evaluation criteria in the Cochrane Handbook, and meta-analysis was performed using RevMan 5.3. Results: Twenty-six literatures that met the criteria, involving 2,174 patients, were included; the meta-analysis showed that compared with western medicine, the use of acupuncture can improve the overall effective rate $(\mathrm{RR}=1.24,95 \% \mathrm{CI}[1.17,1.32], \mathrm{P}<0.00001)$ and reduce the VAS score (MD $=-1.43,95 \%$ CI [-1.97, -0.89], P $<0.00001)$. Conclusion: The use of acupuncture can further improve the clinical effect of patients with postherpetic neuralgia.
\end{abstract}

Keywords: Acupuncture; Postherpetic neuralgia; Meta-analysis; Clinical effect; VAS score

Online publication: January 19, 2022

\section{Introduction}

Herpes zoster is a common skin disease seen in clinical practice. It is an acute infectious skin disease caused by varicella-zoster virus. Herpes zoster usually occurs on one side of the patient's body and generally does not exceed the median line, with pain as the main manifestation. Postherpetic neuralgia is one of the most common complications of herpes zoster. It is a pathologic neuropathic pain that persists after the onset of herpes zoster or remains after the resolution of the herpes zoster. This long-term sequela of herpes zoster has a serious impact on the physical, psychological, daily life, and activity of patients. With age, the incidence of postherpetic neuralgia increases. The incidence of postherpetic neuralgia is about $65 \%$ in patients 60 years of age and older and up to $75 \%$ in those 70 years of age and older ${ }^{[1]}$. For the treatment of postherpetic neuralgia, on the basis of inhibiting virus and nourishing nerves, traditional Chinese medicine (TCM) has put forward the therapeutic methods of relieving collaterals and pain, replenishing qi and nourishing yin, as well as comprehensive repairing. Among them, acupuncture has good therapeutic effect in the treatment of postherpetic neuralgia. Acupuncture can reduce the vascular permeability around inflammatory lesions, inhibit the exudation of inflammatory substances, promote the excretion of inflammatory substances, and at the same time, it has the effect of analgesia and relieving collaterals. It has significant clinical efficacy in improving pain ${ }^{[2]}$. A meta-analysis and systematic review of the clinical 
trials of acupuncture in the treatment of patients with postherpetic neuralgia have been conducted, in order to systematically analyze the therapeutic effect of acupuncture on postherpetic neuralgia, thus providing more evidence-based data for clinical acupuncture treatment in postherpetic neuralgia.

\section{Data and methods}

\subsection{Literature retrieval strategy}

Based on the keyword retrieval method, China National Knowledge Infrastructure (CNKI, 1978-2020), Chinese Biomedical Literature database (CBM, 1979-2020), Chongqing VIP Full-Text Journal Database (WeiPu, 1989-2020), Wanfang Data Knowledge Service Platform (WanFang, 1989-2020), Cochrane Library, PubMed, and Embase were searched. All searches were performed as of December 31, 2020. In order to avoid missing out literatures, "acupuncture," "fire needle," "electroacupuncture," "filiform needle," "herpes zoster," "postherpetic neuralgia," "herpes zoster sequelae," "PHN," and other keywords were used in the search.

\subsection{Inclusion criteria}

The inclusion criteria were as follows: (1) clinical randomized controlled trials; (2) the clinical manifestations, characteristics of the pain, diagnostic criteria, as well as the definitions of cure rate and total effective rate meet the description in the Chinese Expert Consensus on the Diagnosis and Treatment of Postherpetic Neuralgia ${ }^{[1]}$; (3) electroacupuncture, fire acupuncture, and filiform needle are assumed under the category of acupuncture therapy.

\subsection{Exclusion criteria}

The exclusion criteria were as follows: (1) repeatedly published or detected literature data; (2) in the study group or the control group, the treatment regimen adopted multiple combined interventions; although acupuncture therapy is used, it cannot be judged whether acupuncture therapy plays a role in the clinical treatment; (3) trials involving common interventions, such as moxibustion, qigong, massage, and cupping; (4) patients with other serious diseases, such as severe hypertension, severe diabetes, coronary heart disease, and other serious diseases.

\subsection{Outcome indicators}

The primary outcome indicators were the overall response rate of clinical efficacy and visual analogue scale (VAS) score. The secondary outcome indicators were cure rate, recurrence rate, and adverse reaction rate.

\subsection{Literature screening and data extraction}

Uniform inclusion criteria were used. Two investigators independently read the title and abstract of the literatures, excluded those that did not meet the inclusion criteria, and further read the full text to determine whether the inclusion criteria were met. The third person was responsible for the re-evaluation and the inclusion of literatures upon consultation if any inconsistencies occurred. The extraction of data included the first author's name, publication year, sample size of included patients, interventions, outcome measures, etc.

\subsection{Assessment of methodological quality}

The included literatures were assessed according to the risk of bias assessment tool, found in the Cochrane 
Handbook 5.1.0, in terms of the methods (random sequence generation and allocation concealment), whether the blinding of investigators, subjects, and outcome assessors was implemented, the completeness of the outcome data, the occurrence of selective reporting of the results, the methodological quality of each study in 7 areas, as well as the presence or absence of other biases.

\subsection{Statistical analysis}

Meta-analysis was performed on all data using RevMan 5.3. The risk ratio (RR) was used for dichotomous variables and the mean difference (MD) was used as an effect indicator for continuous variables; $95 \%$ confidence interval (CI) was calculated for both. When $\mathrm{P}>0.10$ and $\mathrm{I}^{2}<50 \%$, the heterogeneity between studies was considered small, and the fixed effects model was used; otherwise, the random effects model was used, and sensitivity analysis was performed to find the source of heterogeneity; when necessitated, subgroup analysis was performed to clarify whether clinical and methodological heterogeneity existed.

\section{Results}

\subsection{Literature retrieval and screening process}

A total of 681 literatures were obtained through the literature search. According to the inclusion and exclusion criteria, duplicated literatures without raw data were excluded, and 26 literatures ${ }^{[3-28]}$ were finally included. The literature screening process and results are shown in Figure 1.

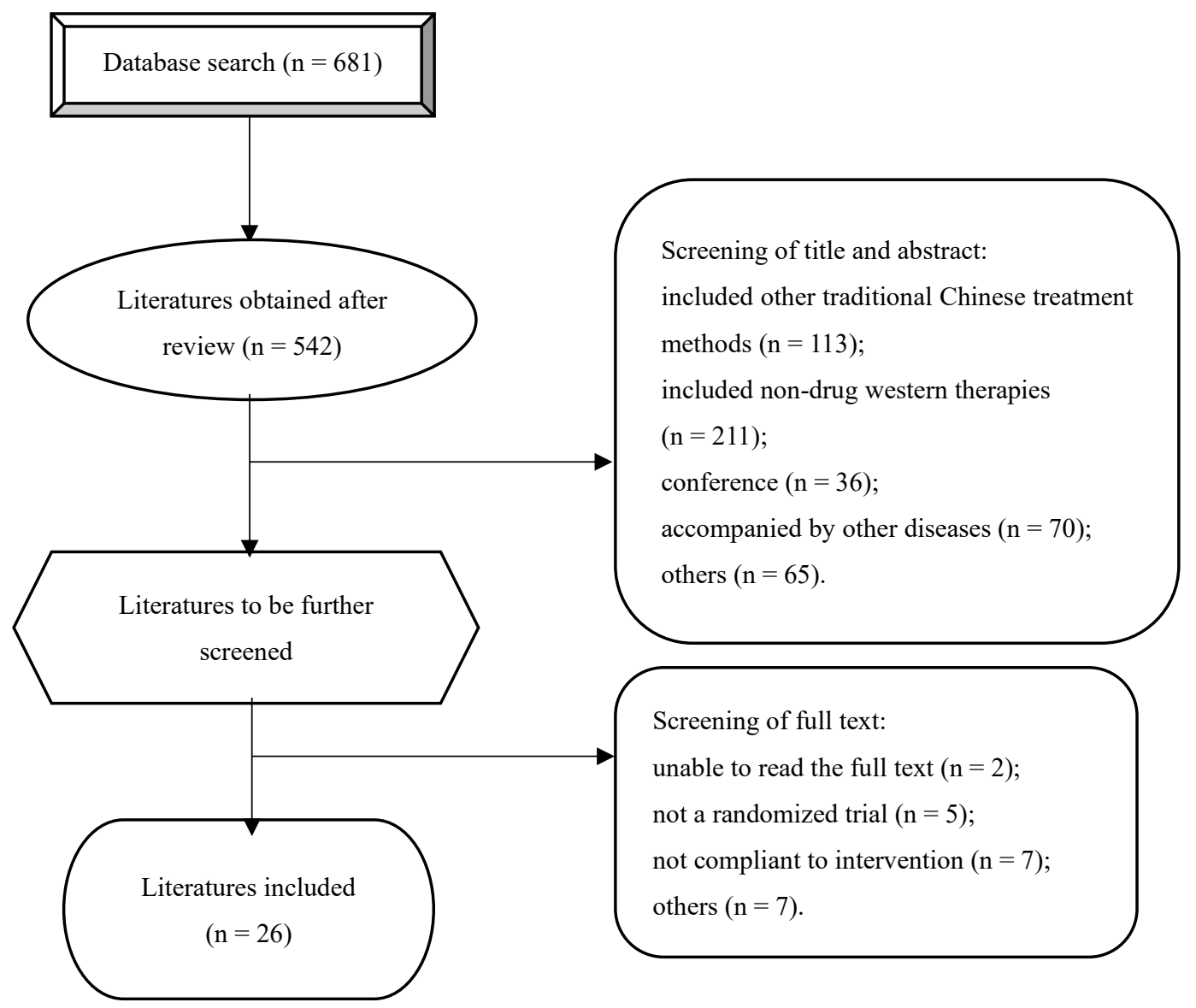

Figure 1. Literature screening process 


\subsection{Basic characteristics of the included literatures}

The total sample size of the 26 trials included was 2,174, including 1,090 in the observation group and 1,082 in the control group. Acupuncture interventions were noted in all 26 trials with clear outcome measures, as shown in Table 1. For the observation group, acupuncture alone was used in 14 trials, acupuncture combined with western medicine was used in 10 trials, and acupuncture combined with traditional Chinese medicine was used in 2 trials. For the control group, western medicine alone was used in 24 trials and traditional Chinese medicine was used in 2 trials. All 26 trials reported the therapeutic effect of acupuncture on postherpetic neuralgia. In terms of outcome measures, 20 trials ${ }^{[3-20,27-28]}$ described using the overall response rate of clinical efficacy, while 17 trials ${ }^{[5-7,10,11,13,14,16,18,20-26,28]}$ described using VAS scores; the recurrence rate was reflected in 4 trials ${ }^{[5,7,14,26]}$, cure rate in 8 trials ${ }^{[6,9,11,13,16,19,20,27]}$, and adverse reaction rate in 4 trials ${ }^{[17,19,22,23]}$.

Table 1. Basic characteristics of the included literatures

\begin{tabular}{|c|c|c|c|c|c|c|}
\hline \multirow{2}{*}{ Literatures } & \multirow{2}{*}{ Year } & \multirow{2}{*}{$\begin{array}{c}\text { Sample size } \\
(\mathrm{O} / \mathrm{C})\end{array}$} & \multirow{2}{*}{ Study type } & \multicolumn{2}{|c|}{ Intervention } & \multirow{2}{*}{ Outcome measures } \\
\hline & & & & Observation group & Control group & \\
\hline Zeng $\mathrm{F}^{[3]}$ & 2016 & $50 / 50$ & Randomized controlled & Acupuncture & Western medicine & Overall response rate \\
\hline Duan $C^{[4]}$ & 2018 & $31 / 31$ & Randomized controlled & Acupuncture & Western medicine & Overall response rate \\
\hline $\mathrm{Li} \mathrm{W}^{[5]}$ & 2018 & $42 / 42$ & Randomized controlled & Acupuncture & Western medicine & $\begin{array}{c}\text { Overall response rate; } \\
\text { VAS score; recurrence rate }\end{array}$ \\
\hline Liang $X^{[6]}$ & 2017 & $60 / 60$ & Randomized controlled & Acupuncture & Western medicine & $\begin{array}{l}\text { Overall response rate; } \\
\text { VAS score; cure rate }\end{array}$ \\
\hline $\operatorname{Lin} S^{[7]}$ & 2020 & $39 / 39$ & Randomized controlled & icture & Western medicine & $\begin{array}{c}\text { Overall response rate; } \\
\text { VAS score; recurrence rate }\end{array}$ \\
\hline $\mathrm{Lu} \mathrm{L}^{[8]}$ & 2020 & $70 / 70$ & Randomized controlled & Acupuncture & Western medicine & Overall response rate \\
\hline Shi A ${ }^{[9]}$ & 2018 & $51 / 51$ & Randomized controlled & Acupuncture & Western medicine & $\begin{array}{l}\text { Cure rate; overall } \\
\text { response rate }\end{array}$ \\
\hline Zhang S [10] & 2020 & $50 / 50$ & Randomiz & ture & Weste & $\begin{array}{c}\text { Overall response rate; } \\
\text { VAS score }\end{array}$ \\
\hline Zheng Y ${ }^{[11]}$ & 2019 & $34 / 34$ & Randomized controlled & Acupuncture & Western medicine & $\begin{array}{c}\text { Cure rate; overall response } \\
\text { rate;VAS score }\end{array}$ \\
\hline Cui W [12] & 2019 & $30 / 30$ & Randomized controlled & $\begin{array}{c}\text { Acupuncture + } \\
\text { western medicine }\end{array}$ & Western medicine & Overall response rate \\
\hline Huang Y [13] & 2019 & $35 / 35$ & Randomized controlled & $\begin{array}{c}\text { Acupuncture + } \\
\text { western medicine }\end{array}$ & Western medicine & $\begin{array}{l}\text { Cure rate; VAS score; } \\
\text { overall response rate }\end{array}$ \\
\hline Li J [14] & 2019 & $35 / 35$ & Randomized controlled & Western medicine & Western medicine & $\begin{array}{c}\text { Overall response rate; } \\
\text { VAS score; recurrence rate }\end{array}$ \\
\hline Wang $\mathbf{J}[15]$ & 2019 & $43 / 43$ & Randomized controlled & $\begin{array}{c}\text { Acupuncture + } \\
\text { western medicine }\end{array}$ & Western medicine & Overall response rate \\
\hline Wang J [16] & 2020 & $45 / 44$ & Randomized controlled & $\begin{array}{c}\text { Acupuncture + } \\
\text { western medicine }\end{array}$ & Western medicine & $\begin{array}{l}\text { Overall response rate; } \\
\text { cure rate; VAS score }\end{array}$ \\
\hline Wang L ${ }^{[17]}$ & 2018 & $30 / 30$ & Randomized controlled & $\begin{array}{c}\text { Acupuncture + } \\
\text { western medicine }\end{array}$ & Western medicine & $\begin{array}{l}\text { Overall response rate; } \\
\text { adverse reaction rate }\end{array}$ \\
\hline Zhao W ${ }^{[18]}$ & 2018 & $29 / 29$ & Randomized controlled & $\begin{array}{c}\text { Acupuncture + } \\
\text { western medicine }\end{array}$ & Western medicine & $\begin{array}{c}\text { Overall response rate; } \\
\text { VAS score }\end{array}$ \\
\hline
\end{tabular}

(Continued on the next page) 
(Continued from previous page)

\begin{tabular}{|c|c|c|c|c|c|c|}
\hline \multirow{2}{*}{ Literatures } & \multirow{2}{*}{ Year } & \multirow{2}{*}{$\begin{array}{l}\text { Sample size } \\
(\mathrm{O} / \mathrm{C})\end{array}$} & \multirow{2}{*}{ Study type } & \multicolumn{2}{|c|}{ Intervention } & \multirow{2}{*}{ Outcome measures } \\
\hline & & & & Observation group & Control group & \\
\hline $\operatorname{Li} Z^{[19]}$ & 2018 & $30 / 30$ & Randomized controlled & $\begin{array}{l}\text { Acupuncture + } \\
\text { TCM }\end{array}$ & TCM & $\begin{array}{l}\text { Cure rate; overall response } \\
\text { rate; adverse reaction rate }\end{array}$ \\
\hline Wang C ${ }^{[20]}$ & 2020 & $30 / 30$ & Randomized controlled & $\begin{array}{c}\text { Acupuncture + } \\
\text { TCM }\end{array}$ & TCM & $\begin{array}{l}\text { Cure rate; overall } \\
\text { response rate; VAS score }\end{array}$ \\
\hline Gong $\mathrm{Y}^{[21]}$ & 2018 & $32 / 32$ & Randomized controlled & Acupuncture & Western medicine & VAS score \\
\hline Huo $X^{[22]}$ & 2020 & $35 / 35$ & Randomized controlled & Acupuncture & Western medicine & $\begin{array}{l}\text { VAS score; adverse } \\
\text { reaction rate }\end{array}$ \\
\hline Wu C [23] & 2018 & $26 / 26$ & Randomized controlled & Acupuncture & Western medicine & $\begin{array}{l}\text { VAS score; adverse } \\
\text { reaction rate }\end{array}$ \\
\hline Zhang Y [24] & 2018 & $40 / 40$ & Randomized controlled & Acupuncture & Western medicine & VAS score \\
\hline Luo Y ${ }^{[25]}$ & 2019 & $49 / 49$ & Randomized controlled & $\begin{array}{c}\text { Acupuncture }+ \\
\text { western medicine }\end{array}$ & Western medicine & VAS score \\
\hline Wang Y [26] & 2016 & $39 / 39$ & Randomized controlled & $\begin{array}{c}\text { Acupuncture }+ \\
\text { western medicine }\end{array}$ & Western medicine & VAS score; recurrence rate \\
\hline Fang $X^{[27]}$ & 2013 & $107 / 104$ & Randomized controlled & Acupuncture & Western medicine & $\begin{array}{l}\text { Overall response rate; } \\
\text { cure rate }\end{array}$ \\
\hline Zhang M ${ }^{[28]}$ & 2014 & $28 / 26$ & Randomized controlled & $\begin{array}{c}\text { Acupuncture }+ \\
\text { western medicine }\end{array}$ & Western medicine & $\begin{array}{c}\text { Overall response rate; } \\
\text { VAS score }\end{array}$ \\
\hline
\end{tabular}

\subsection{Quality assessment of the included literatures}

The quality assessment of the included literatures was evaluated with reference to Cochrane's Bias Risk

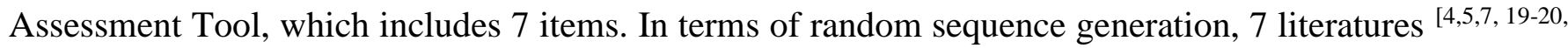
${ }^{24,25]}$ used the random number table method, 1 trial ${ }^{[18]}$ used the digital parity grouping method, and the remaining 18 literatures did not specify any specific randomization method. There was no statistically significant difference $(\mathrm{P}>0.05)$ in the evaluation results of the general data (age, disease duration, onset time, and severity) between the observation groups and the control groups ( $\mathrm{P}>0.05)$. According to the risk of bias assessment, only 1 article ${ }^{[18]}$ was high risk, while the rest were low risk. In terms of the hidden aspects of random assignment, none of the 26 literatures were unclear. In terms of implementer and subject blinding, only 1 article ${ }^{[4]}$ was double-blinded, while the remaining 25 literatures ${ }^{[3-5,7-28]}$ did not mention anything related to it, which were low risk. In terms of blinding of outcome assessment, none of the 26 literatures mentioned it as unclear, while in terms of data integrity and selectivity, with reference to the study protocol designed in the literatures, the data and results reported in the literatures were complete and low risk. Other biases were not mentioned in any of the included literatures and were considered low risk. The evaluation results are shown in Figure 2. 


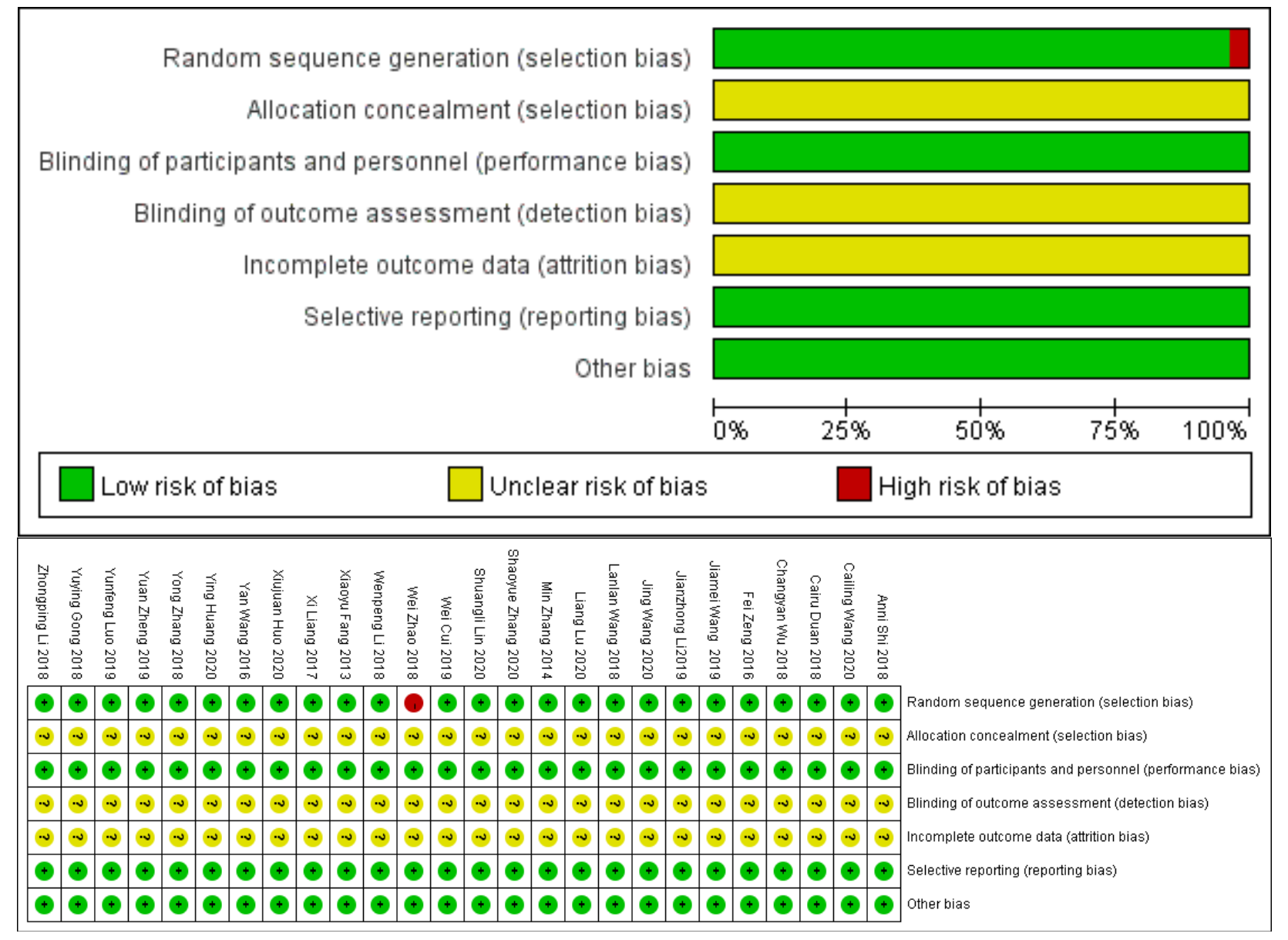

Figure 2. Risk of bias assessment of the included literatures

\subsection{Meta-analysis}

\subsubsection{Overall effective rate}

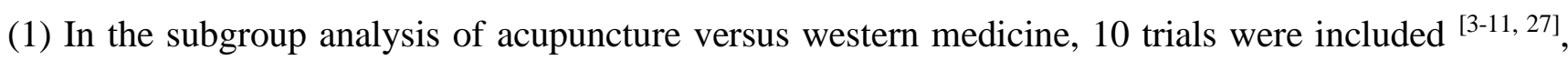
with a total of 1,065 subjects. There was no heterogeneity $\left(\mathrm{P}=0.30, \mathrm{I}^{2}=16 \%\right)$ among the studies, and the fixedeffects model was used. The results showed that the overall effective rate of the observation group was higher than that of the control group, with a significant statistical difference $(\mathrm{RR}=1.24,95 \% \mathrm{CI}[1.17,1.32], \mathrm{P}<0.00001)$, as shown in Figure 3. It is suggested that acupuncture can further improve the overall effective rate of patients with postherpetic neuralgia.

(2) In the subgroup analysis of acupuncture combined with western medicine compared with western medicine, 8 trials were included ${ }^{[12-18,28]}$, with a total of 547 subjects. There was no heterogeneity (P $=0.47, \mathrm{I}^{2}=0 \%$ ) among the studies, and the fixed effects model was used. The results showed that the overall effective rate of the observation group was higher than that of the control group, with a significant statistical difference $(\mathrm{RR}=1.20,95 \% \mathrm{CI}[1.12,1.29], \mathrm{P}<0.00001)$, as shown in Figure 3. It is suggested that acupuncture can effectively increase the overall effective rate of patients with postherpetic neuralgia on the basis of using western medicine.

(3) In the subgroup analysis of acupuncture combined with traditional Chinese medicine compared with traditional Chinese medicine only, a total of 120 subjects were included in 2 trials ${ }^{[19,20]}$. There was no heterogeneity $\left(\mathrm{P}=1.00, \mathrm{I}^{2}=0 \%\right)$ between the studies, and the fixed effects model was used. The results showed that the overall effective rate of the observation group was higher than that of the 
control group, with a significant statistical difference $(\mathrm{RR}=1.32,95 \% \mathrm{CI}[1.12,1.55], \mathrm{P}=0.0007)$, as shown in Figure 3. It is suggested that acupuncture can significantly improve the overall effective rate of patients with postherpetic neuralgia on the basis of using traditional Chinese medicine.

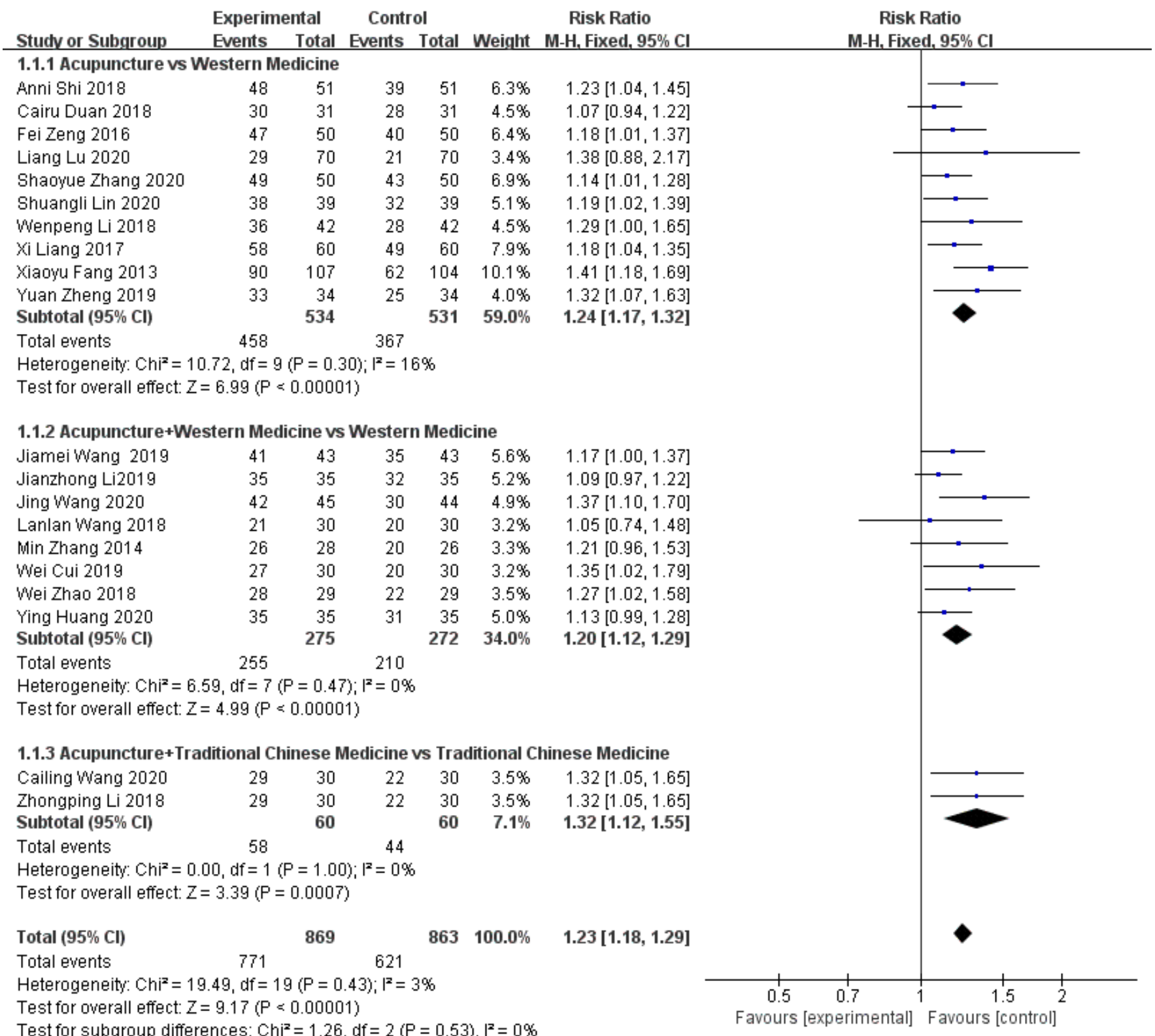

Figure 3. Forest plot for overall response rate

\subsubsection{VAS score}

(1) In the subgroup analysis of acupuncture versus western medicine, 9 trials were included ${ }^{[5-7,10,11,21-}$ ${ }^{24]}$, with a total of 690 subjects. There was heterogeneity among the studies $\left(\mathrm{P}<0.00001, \mathrm{I}^{2}=99 \%\right)$, and the random effects model was used. The results showed that the VAS score of the observation group was lower than that of the control group, with a significant statistical difference (MD $=-1.43$, 95\% CI [-1.97, -0.89], $\mathrm{P}<0.00001)$, as shown in Figure 4. It is suggested that acupuncture can further reduce the VAS score of patients with postherpetic neuralgia. 
(2) In the subgroup analysis of acupuncture combined with western medicine compared with western medicine alone, 7 trials were included ${ }^{[13,14,16,18,25,26,28]}$, with a total of 517 subjects. There was heterogeneity among the studies $\left(\mathrm{P}<0.00001, \mathrm{I}^{2}=89 \%\right)$, and the random effects model was used. The results showed that the VAS score of the observation group was lower than that of the control group, with a significant statistical difference (MD $=-1.87,95 \%$ CI [-2.35, -1.40], P < 0.00001), as shown in Figure 4. It is suggested that acupuncture can effectively reduce the VAS score of patients with postherpetic neuralgia on the basis of western medicine.

(3) In the subgroup analysis of acupuncture combined with traditional Chinese medicine compared with traditional Chinese medicine alone, only 1 trial was included ${ }^{[20]}$, with a total of 60 subjects. There is insufficient evidence that acupuncture combined with traditional Chinese medicine can effectively reduce the VAS score of patients with postherpetic neuralgia.

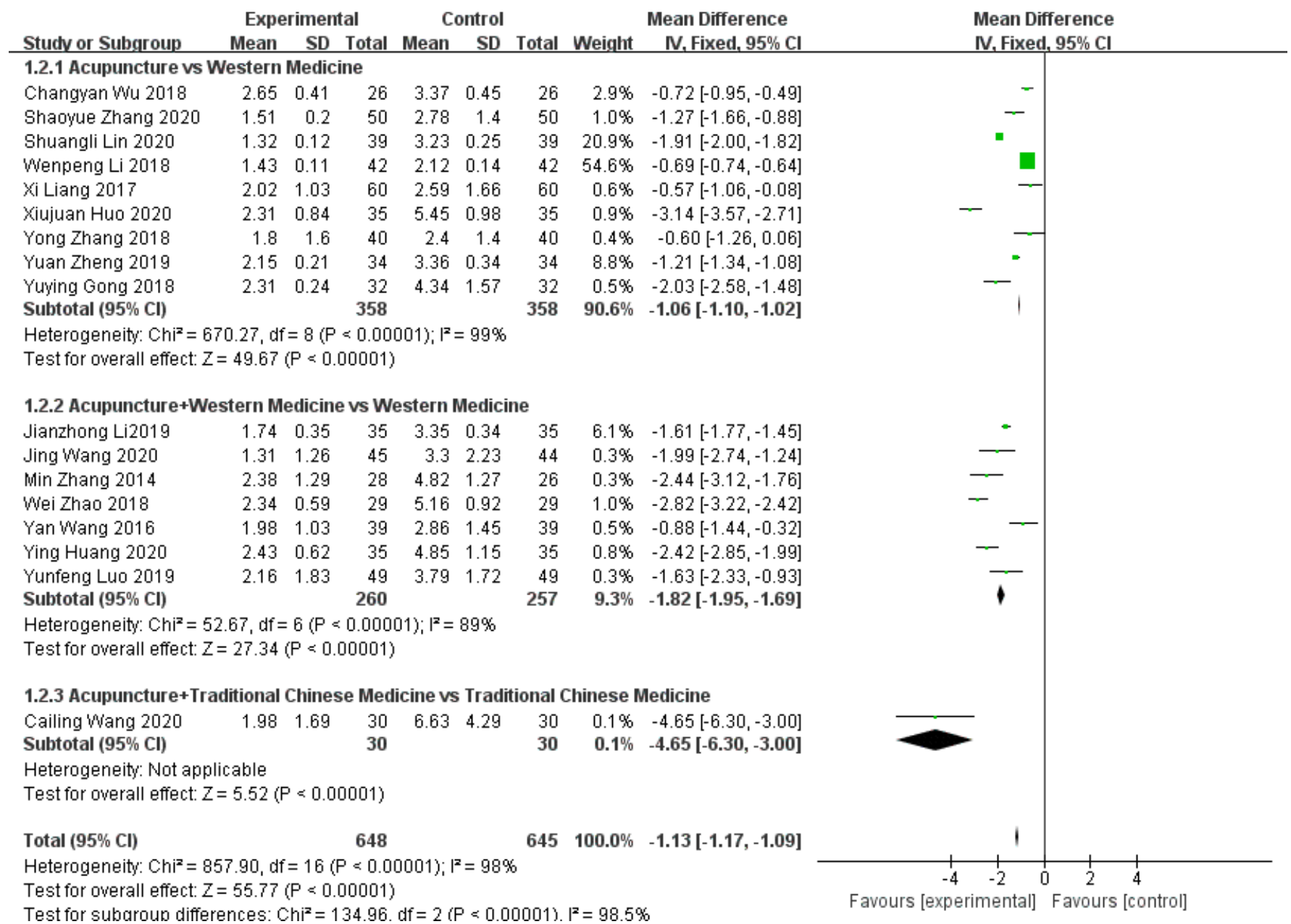

Figure 4. Forest plot for VAS score

\subsubsection{Cure rate}

(1) In the subgroup analysis of acupuncture compared with western medicine, 4 trials reported on cure rate $^{[6,9,11,27]}$, with a total of 501 subjects. There was no heterogeneity $\left(\mathrm{P}=0.30, \mathrm{I}^{2}=18 \%\right)$ among the studies, and the fixed effects model was used. The results showed that the cure rate of the observation group was higher than that of the control group, with a significant statistical difference ( $R R=2.17$, 95\% CI [1.59, 2.98], $\mathrm{P}<0.00001)$, as shown in Figure 5. It is suggested that acupuncture can further improve the cure rate of patients with postherpetic neuralgia. 
(2) In the subgroup analysis of acupuncture combined with western medicine compared with western medicine alone, 2 trials were included ${ }^{[13,15]}$, with a total of 159 subjects. There was no heterogeneity $(\mathrm{P}=0.92, \mathrm{I} 2=0 \%)$ between the studies, and the fixed effects model was used. The results showed that the cure rate of the observation group was higher than that of the control group, with a significant statistical difference $(\mathrm{RR}=1.73,95 \%$ CI $[1.19,2.51], \mathrm{P}=0.004)$, as shown in Figure 5. It is suggested that acupuncture can effectively improve the cure rate of patients with postherpetic neuralgia on the basis of western medicine.

(3) In the subgroup analysis of acupuncture combined with traditional Chinese medicine compared with traditional Chinese medicine alone, 2 trials reported on cure rate ${ }^{[19,20]}$, with a total of 120 subjects. There was no heterogeneity $\left(\mathrm{P}=0.89, \mathrm{I}^{2}=0 \%\right)$ between the studies, and the fixed effects model was used. The results showed that the cure rate of the observation group was higher than that of the control group, with a significant statistical difference $(\mathrm{RR}=2.60,95 \% \mathrm{CI}[1.38,4.89], \mathrm{P}=0.003)$, as shown in Figure 5. It is suggested that acupuncture can significantly improve the cure rate of patients with postherpetic neuralgia on the basis of traditional Chinese medicine.

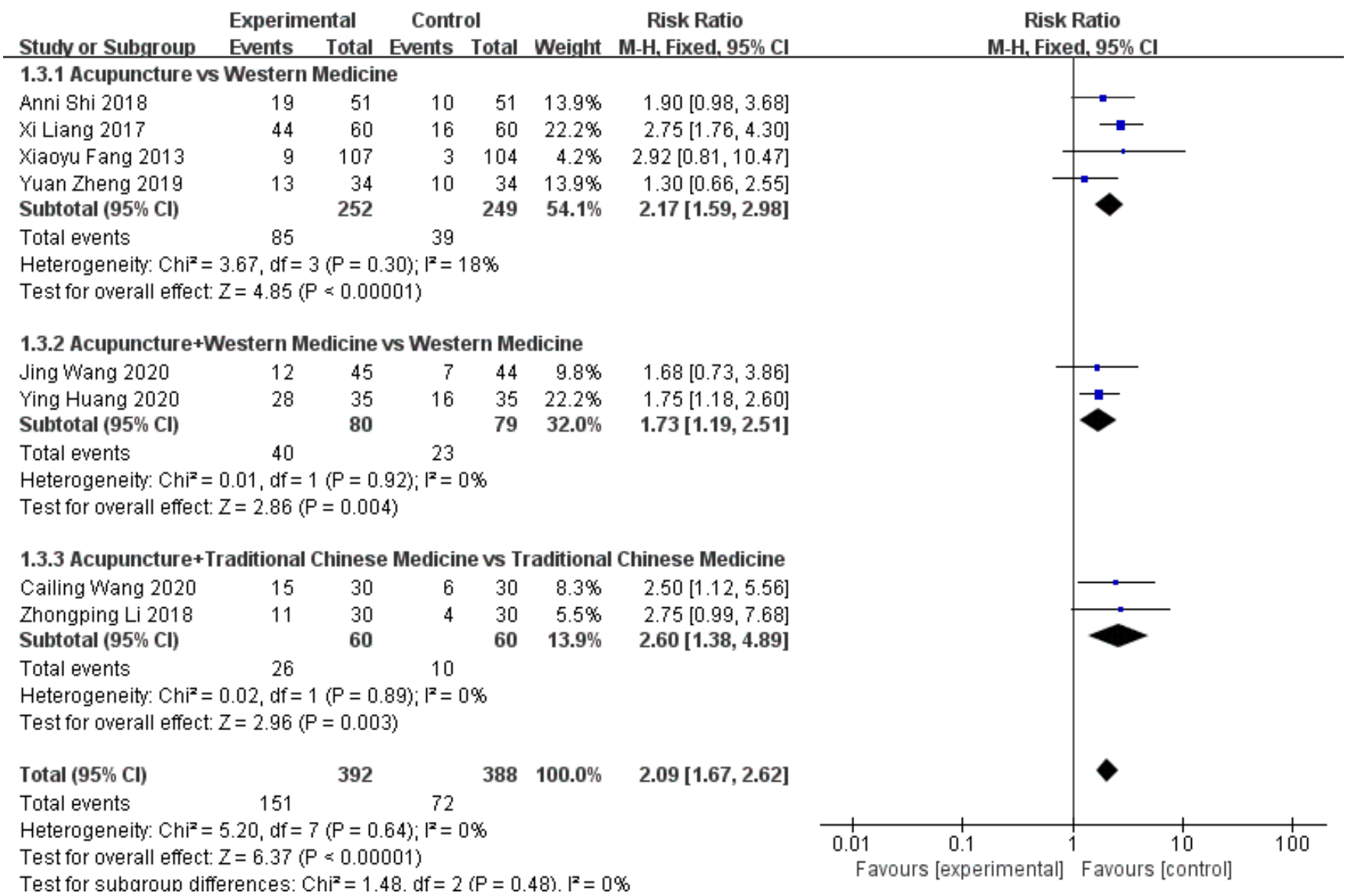

Figure 5. Forest plot for cure rate

\subsubsection{Recurrence rate}

(1) In the subgroup analysis of acupuncture versus western medicine, a total of 162 subjects were included in 2 trials ${ }^{[5,7]}$. There was no heterogeneity $\left(\mathrm{P}=0.31, \mathrm{I}^{2}=3 \%\right)$ between the studies, and the fixed effects model was used. The results showed that the recurrence rate of the observation group was lower than that of the control group, with a significant statistical difference $(\mathrm{RR}=0.43,95 \% \mathrm{CI}$ 
$[0.17,1.06], \mathrm{P}=0.07)$, as shown in Figure 6. It is suggested that acupuncture can reduce the recurrence rate of patients with postherpetic neuralgia.

(2) In the subgroup analysis of acupuncture combined with western medicine compared with western medicine alone, 2 trials reported on recurrence rate ${ }^{[14,26]}$, with a total of 148 subjects. There was no heterogeneity $\left(\mathrm{P}=0.12, \mathrm{I}^{2}=60 \%\right)$ between the studies, and the fixed effects model was used. The results showed that the recurrence rate of the observation group was lower than that of the control group, with a significant statistical difference $(\mathrm{RR}=0.33,95 \% \mathrm{CI}[0.05,1.97], \mathrm{P}=0.22)$, as shown in Figure 6. It is suggested that acupuncture can effectively reduce the recurrence rate of patients with postherpetic neuralgia on the basis of western medicine.

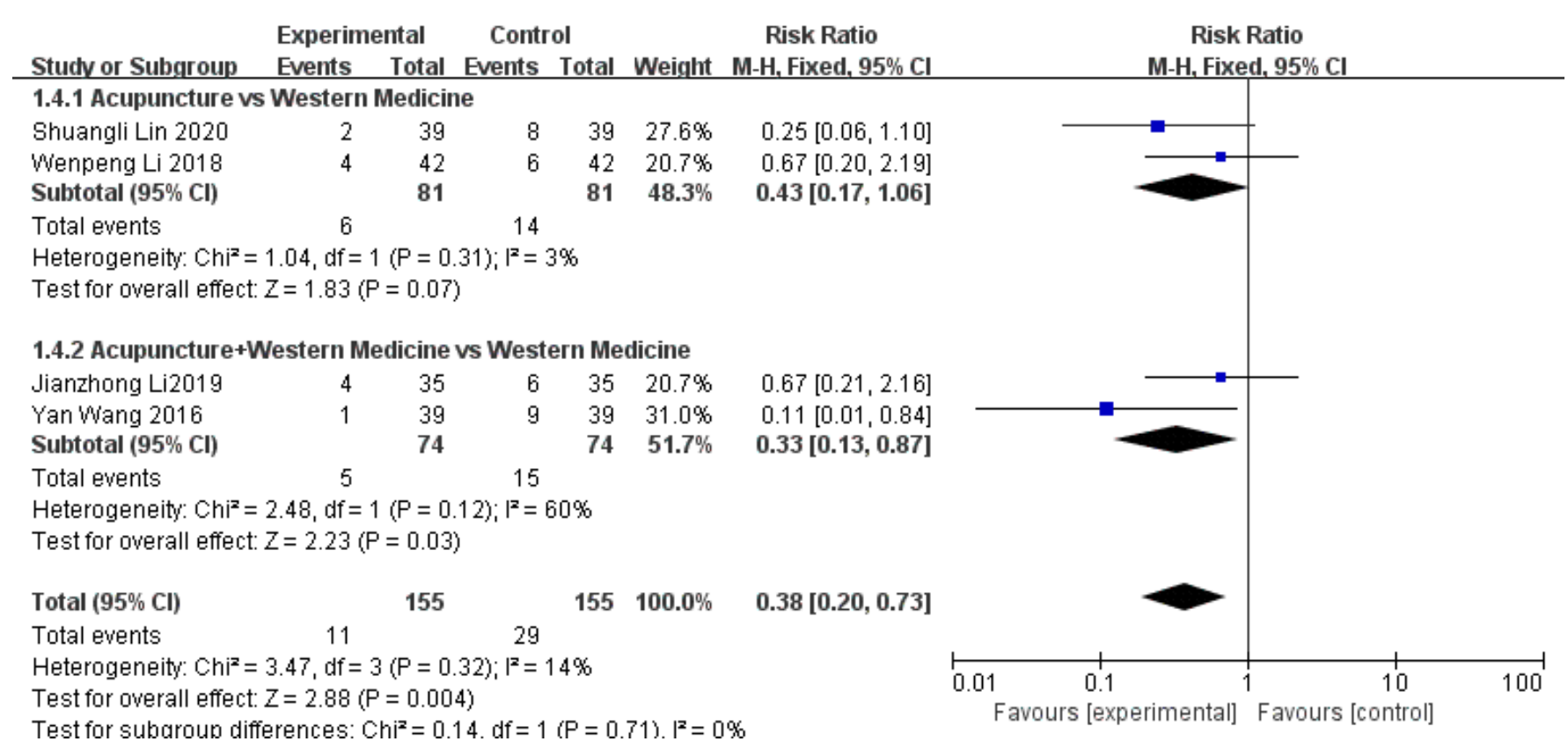

Figure 6. Forest plot for recurrence rate

\subsubsection{Adverse reaction rate}

(1) In the subgroup analysis of acupuncture compared with western medicine, a total of 124 subjects were included in 2 trials ${ }^{[22,23]}$. There was no heterogeneity $\left(\mathrm{P}=0.54, \mathrm{I}^{2}=0 \%\right)$ between the studies, and the fixed effects model was used. The results showed that the adverse reaction rate of the observation group was lower than that of the control group, with a significant statistical difference $(\mathrm{RR}=0.20,95 \% \mathrm{CI}[0.04,1.11], \mathrm{P}=0.07)$, as shown in Figure 7. It is suggested that acupuncture can further reduce the adverse reactions of patients with postherpetic neuralgia.

(2) In the subgroup analysis of acupuncture combined with traditional Chinese medicine compared with traditional Chinese medicine alone, only 1 trial was included ${ }^{[19]}$, and a total of 60 subjects. Therefore, there is insufficient evidence that acupuncture combined with traditional Chinese medicine can reduce the rate of adverse reaction in patients with postherpetic neuralgia. 


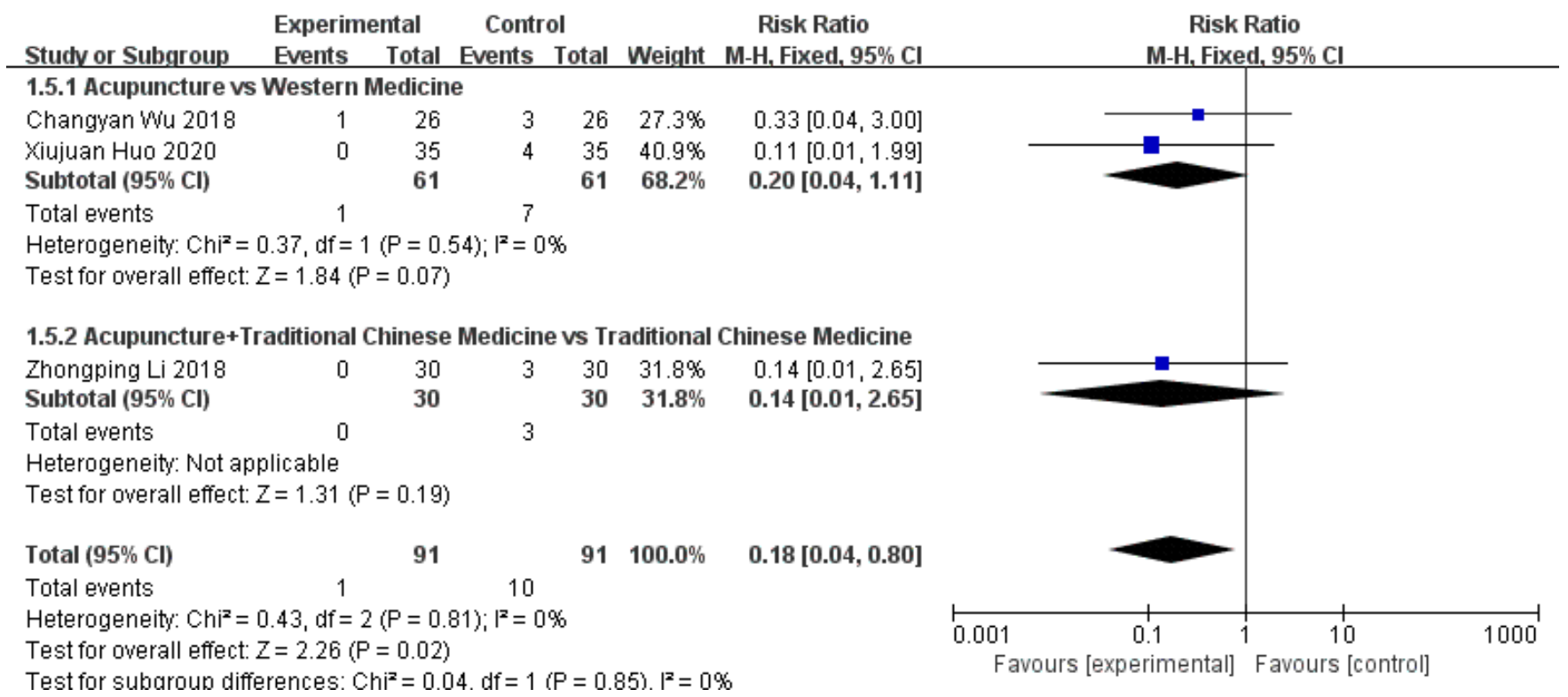

Figure 7. Forest plot for adverse reaction rate

\section{Discussion}

Herpes zoster is mainly caused by varicella-zoster virus infection. Postherpetic neuralgia is one of the main complications of herpes zoster. The pain experienced by patients affected by postherpetic neuralgia can seriously affect their life and activity. Therefore, timely and effective treatment is necessary. The disease is mainly restricted by advanced age, late treatment, short course of treatment, other primary diseases, and other factors. Pharmacological studies have shown that in the routine symptomatic supportive treatment, the combination of multiple drugs, leading to various adverse reactions, such as amenorrhea, impotence, delayed dyskinesia, dry mouth, constipation, liver function derangement, granulocytopenia, etc., would bring more pain to the patients and affect their compliance to treatment.

In traditional Chinese medicine, it is believed that postherpetic neuralgia is related to a deficiency of vital energy, disturbance of Ying and defense, as well as incompatibility of meridians and collaterals. Acupoint selection based on syndrome differentiation and acupuncture at meridian points can gradually relieve the pain of patients. The results from this meta-analysis showed that in the treatment of postherpetic neuralgia, comparing acupuncture with western medicine, the use of acupuncture can further improve the overall effective rate $(\mathrm{RR}=1.24,95 \% \mathrm{CI}[1.17,1.32], \mathrm{P}<0.00001)$ and reduce the VAS score of patients $(\mathrm{MD}=-1.43,95 \% \mathrm{CI}[-1.97,-0.89], \mathrm{P}<0.00001)$. The method is simple and can effectively improve the pain as well as the quality of life of patients suffering from postherpetic neuralgia.

However, there are some limitations in this study. Through literature collocation, it was noted that the sample size of the literatures reporting about the use of acupuncture in the treatment of postherpetic neuralgia is relatively small, double-blinded clinical trials are insufficient, the records of loss to follow-up, withdrawal, and adverse events occurring in the clinical trials were not detailed enough, and the late followup records were incomplete. As a result, partial data were not included in the analysis, which may affect the reliability of the results. It is hoped that there will be more high-quality literatures in future studies to clarify the clinical effect of acupuncture in comparison with other therapeutic methods, provide more valuable references for future scientific research and clinical practice, as well as add to the development of the TCM cause. 


\section{Disclosure statement}

The authors declare that there is no conflict of interest.

\section{References}

[1] Yu S, Wan Y, Wang Q, et al., 2016, Chinese Expert Consensus on the Diagnosis and Treatment of Postherpetic Neuralgia. China Pain Medicine Miscellaneous, 22(3): 161-167.

[2] Chen H, Chen J, Yang Y, et al., 2009, Clinical Observation of Different Acupuncture Methods in the Treatment of Postherpetic Neuralgia. Acupuncture Clinical Journal, 25(4): 31-33.

[3] Zeng F, 2016, Clinical Effect Analysis of Chinese Medicine Acupuncture in the Treatment of Postherpetic Neuralgia. World Abstract of the Latest Medical Information, 16(05): 134.

[4] Duan C, 2018, Clinical Observation of Acupuncture-Based Treatment of Postherpetic Neuralgia. Guangming TCM, 33(5): 681-683.

[5] Li W, 2018, Clinical Effect Analysis of Chinese Medicine Acupuncture for Postherpetic Neuralgia. Primary Medical Forum, 22(26): 3731-3732.

[6] Liang X, 2017, Clinical Effect Observation of Chinese Medicine Acupuncture in the Treatment of Postherpetic Neuralgia. Health Care Guide, 2017(41): 49.

[7] Lin S, 2020, Analysis of Clinical Efficacy and Safety of Acupuncture in the Treatment of Postherpetic Neuralgia in Chinese Medicine. Medical Aesthetics and Beauty, 29(12): 87.

[8] Lu L, 2020, Study on the Effect of Chinese Medicine Acupuncture in the Treatment of Postherpetic Neuralgia. Chinese Community Physicians, 36(17): 116-118.

[9] Shih A, 2018, Clinical Effect Analysis of Chinese Medicine Acupuncture for Postherpetic Neuralgia. Diet Science, 2018(20): 118.

[10] Zhang S, 2020, Clinical Observation of Acupuncture for Postherpetic Neuralgia. Guangming TCM, 2020(1): 80-83.

[11] Zheng Y, 2019, Efficacy of the Combination of Acupuncture and Moxibustion Superior Techniques in the Treatment of Postherpetic Neuralgia. World Abstract of The Latest Medical Information, 2019(36): $50,57$.

[12] Cui W, 2019, Exploring the Clinical Effect Observation of Acupuncture for Postherpetic Neuralgia. Diet and Health Care, 6(26): 92.

[13] Huang Y, 2020, Clinical Observation of Fire Acupuncture for Postherpetic Neuralgia. Guangming TCM, 2020(5): 713-715.

[14] Li J, Li J, Si Z, 2019, Observation on the Effect of Acupuncture Rehabilitation for Postherpetic Neuralgia. Frontiers of Medicine, 9(24): 212-213.

[15] Wang J-M, 2019, Study on the Effect of Combined Acupuncture Therapy and Acyclovir in the Treatment of Herpes Zoster. Contemporary Medicine Series, 17(6): 184-185.

[16] Wang J, 2020, Observation on the Effect of Acupuncture in the Treatment of Postherpetic Neuralgia [J]. Frontiers of Medicine, 10(13): 228-229.

[17] Wang L, 2018, Clinical Effects of Acupuncture for Postherpetic Neuralgia. Chinese Practical Medicine, 13(11): 135-136.

[18] Zhao W, 2018, Analysis of the Effect of Acupuncture in the Treatment of Postherpetic Neuralgia. Psychiatrist, 24(24): 160-161. 
[19] Li C, 2018, Clinical Observation of Blood Activation and Blood Dispersion Soup Combined with Fire Acupuncture for Postherpetic Neuralgia. Chinese Journal of Ethnic Folk Medicine, 27(6): 86-87.

[20] Wang C, 2020, Analysis of the Efficacy of Fire Acupuncture with Yi Qi and Blood Activation Tang in the Treatment of Postherpetic Neuralgia with Deficiency of Qi and Blood. Xinjiang Traditional Chinese Medicine, 38(2): 33-35.

[21] Gong Y, 2018, Clinical Effect Analysis of Chinese Medicine Acupuncture for Postherpetic Neuralgia. Medical Food Therapy and Health, 2018(8): 159, 161.

[22] Huo X, 2020, Study on the Clinical Effect of Chinese Medicine Acupuncture in the Treatment of Postherpetic Neuralgia. Health Must Read, 2020(30): 210.

[23] Wu CY, 2018, Clinical Effect Analysis of Acupuncture and Moxibustion for Postherpetic Neuralgia. Inner Mongolia Traditional Chinese Medicine, 37(04): 79.

[24] Zhang Y, Xue Z, Li P, 2018, 40 Cases of Postherpetic Neuralgia Treated with Plum Blossom Acupuncture by Tapping on the Governor's Vein. Journal of Shaanxi University of Traditional Chinese Medicine, 41(6): 85-87.

[25] Luo Y, 2019, Observation on the Effect of Fire Acupuncture Therapy in the Treatment of Postherpetic Neuralgia. Contemporary Medicine Series, 2019(14): 198-199.

[26] Wang Y, 2016, Study on the Clinical Effect of Acupuncture Combined with Drugs for the Treatment of Acute Herpes Zoster and Prevention of Posterior Neuralgia. Chinese Prescription Drugs, 14(9): 9697.

[27] Fang X, 2013, Clinical Efficacy of Electroacupuncture Direct Needling in the Treatment of Postherpetic Neuralgia of Zoster. People's Military Medicine, 56(12): 1427-1428.

[28] Zhang M, 2014, Clinical Observation of 28 Cases of Postherpetic Neuralgia Treated with Needle and Medicine Combination. Chinese Medicine Herald, 20(12): 93-94.

Publisher's note

Bio-Byword Scientific Publishing remains neutral with regard to jurisdictional claims in published maps and institutional affiliations. 